\title{
A Resonant-Mode Model of Pulsar Radio Emission
}

\author{
M. D. T. Young \\ School of Physics, University of Western Australia, 35 Stirling Highway, \\ Crawley WA 6009, Australia
}

\begin{abstract}
It is argued that the polar gap and flux tube in the pulsar magnetosphere act as a resonant cavity/waveguide system which is excited by oscillations in the primary beam current and accelerating potential. The modes will be converted, probably scattered, to produce radio beams in the frequency range of those observed.
\end{abstract}

\section{Introduction}

We live in a resonant cavity bounded below by the earth and above by the ionosphere. Lightning strikes excite low-frequency electromagnetic "Schumann resonances" in this cavity, as predicted by Schumann (1952), with a fundamental frequency of about $8 \mathrm{~Hz}$. Such behavior should also occur above the magnetic poles of pulsars due to the well-defined boundaries found there.

One of these boundaries is the stellar surface which, because of the star's large electron number density, has a conductivity perpendicular to the magnetic field comparable to that of copper; the boundary condition there can be taken to be $E_{\perp}=0$, where perpendicular, $\perp$, and parallel, $\|$, are taken relative to the stellar magnetic field. Second, there is the boundary between the open and closed magnetic field lines, which forms a diverging tube. On the closed field lines there is a non-neutral plasma at the Goldreich-Julian charge density (Goldreich \& Julian 1969), which is co-rotating with the star. The strong stellar magnetic field, $B_{*} \sim 10^{12} \mathrm{G}$, restricts the particle motion to be essentially along the field lines, but this still allows them to respond to an applied $E_{\|}$. The plasma can maintain $E_{\|}=0$ on the tubular boundary at frequencies up to the plasma frequency, which is $\sim(7.5 \mathrm{GHz})\left(B_{*} / 10^{12} \mathrm{G}\right)^{1 / 2}(0.1 \mathrm{~s} / P)^{1 / 2}$ near the stellar surface. On the open field lines particles are accelerated away from the star by a non-zero $E_{\|}$to ultra-relativistic speeds, creating $e^{-} / e^{+}$pairs at a pair-formation front, a third boundary. Beneath the front is the low-density acceleration zone, or polar gap; above it the pairs stream up the tube away from the star forming a dense relativistic secondary plasma. The consequences of a second front forming the gap's base (e.g. Harding \& Muslimov 1998) are also being considered, but will not be discussed here.

\section{Overview of the Model}

Sturrock (1971) noted that the current of particles up the tube might oscillate due to intermittent screening of the accelerating field at the surface of the star 

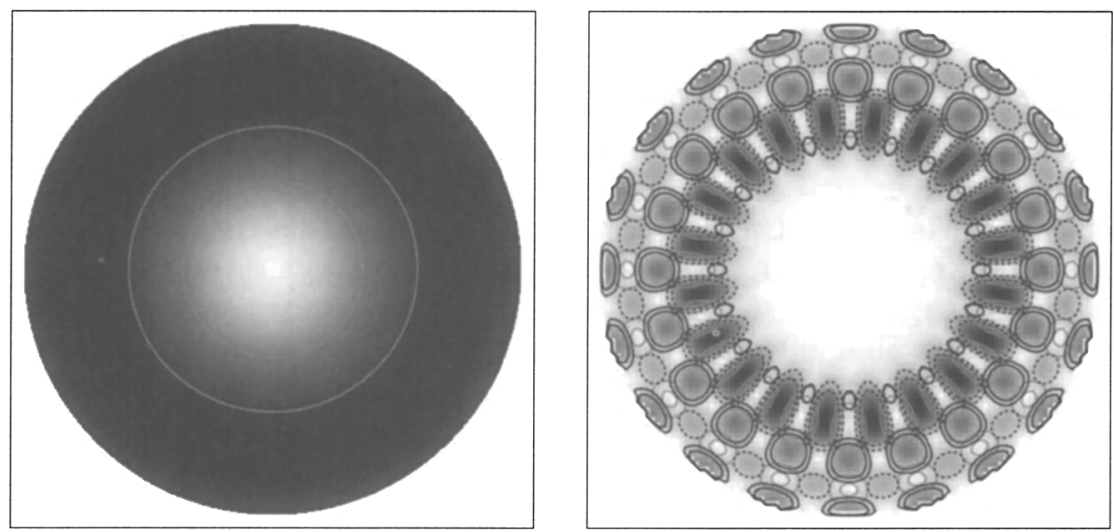

Figure 1. Plots of the energy densities, $U_{\perp}$ and $U_{\|}$, of two modes: left $(m, n)=(0,1)$, right $(m, n)=(10,2)$. The shading shows $U_{\perp}$, with maxima in black. The gray contours delineate regions at the center of which $U_{\|}$is maximum; the black and dashed contours delineate where $E_{\perp}$ has one of two orthogonal polarizations.

by positrons returning from the pair-formation region, and this idea remains plausible. Assume for now that the current has an oscillating component; either this oscillation is in the form of the "sparking" of Ruderman \& Sutherland (1975), or it occurs as an AC component of the DC space-charge limited flow from the surface. This assumption will be revisited below.

The AC component of current/potential will generate large-amplitude electromagnetic waves (Qiao 1988) which will be essentially transverse magnetic $\left(B_{\|} \approx 0\right)$ and have frequencies set by the light travel time across the gap height, $h \sim 10^{2 \pm 1} \mathrm{~m}, \sim 0.1-10 \mathrm{MHz}$. Taking account of the boundary conditions discussed above, the waves will propagate in the tube as waveguide modes. Ignoring stellar rotation and vacuum polarization, and assuming an infinite stellar magnetic field - so that the dielectric tensor of the plasma is diagonal - the tube near the star supports electromagnetic waveguide modes which in flat spacetime are generally well approximated by the transverse magnetic modes of a cylindrical waveguide of arbitrary cross-section. In a circular cylinder the modes are specified by two mode numbers $m$ and $n$, and examples are shown in Figure 1 . Taking account of the change in the dielectric tensor at the pair-formation front, the modes are equally applicable both below and above the front.

The waveguide mode impedances change at the pair-formation front so modes in the gap will be partially reflected from this boundary, and since the modes will also be reflected from the stellar surface, the gap can act as a resonant cavity. I have studied the dispersion relation of the plasmas below and above the front for various configurations and will present my findings elsewhere. Resonances are possible in the gap at frequencies just above the cutoff frequency of a vacuum filled cavity, though the dispersion relation differs from that of the vacuum case. In the secondary plasma above the front there are two or three modes that can carry power up the tube; all have different wavelengths, but 
the same low frequency as the resonance in the gap. Preliminary calculations indicate that the oscillating $E_{\|}$in the wind just above the gap may be many times the oscillating $E_{\|}$in the gap so that, even if the AC component of field in the gap is a small fraction of the DC component, the oscillating electric fields above the gap could be large.

When the driving frequency equals the frequency of a particular mode, and the source oscillations are positioned where that mode has antinodes, then that mode will be excited in the gap. If the source locations were to be confined symmetrically to any particular areas of the polar cap, then we might suppose these to be either near the tube axis, or perhaps close to the wall where a return current may flow. The former would tend to excite the $m=0$ modes, and the latter, high-order $m$ modes.

There are at least two phenomena that may provide a feedback mechanism in the gap. The first is the resonant field itself; energy pumped into the field by the current in turn acts back on the current twice per cycle. Secondly the large $E_{\|}$above the front may accelerate particles of charge opposite to the primary beam current back into the gap; this charge would then cross the gap in time $h / c$ to provide increased screening at the base of the gap. These phenomena would tend to amplify the current/potential oscillations making the gap unstable to resonance, and supporting the assumption made above of an AC component. Also the modes will be coupled via the current, and this raises the possibilities of mode-locking, intermittency and chaos, all of which will be discussed elsewhere.

The plasmas in the tube will posses a spatio-temporal sub-structure, in particular above the antinodes of the resonant modes. Perhaps this structure together with the oscillating fields in the tube go on to produce the observed radio emission via some sort of instability mechanism (see Melrose, these proceedings, for a review). However, preliminary calculations indicate that at least one of the modes in the tube will be scattered to the observed radio frequencies by the inhomogeneities in the wind. With increasing altitude in the tube, the plasma density decreases and the tube diameter increases altering the local dispersion relation, and this determines the range of altitudes in which the scattering can occur.

Several features of pulsar radio emission have a natural interpretation in the model. The stability of mean pulse profiles will be explained if the same modes are continuously excited, or there is rapid switching between a fixed subset of modes. If switching between modes occurs on timescales of several pulses or more, it will be recognized as the observed profile changes. Nulling will be observed if the mode has a low amplitude in the line of sight, or if the resonance (and hence the emission) temporarily ceases - perhaps because the pair-formation fronts become very irregular or the driving oscillations cease as the primary beam current becomes steady. The model can produce short timescale intensity variations due to a few factors, including the inhomogeneity in the plasmas and the finite $Q$-factor of the cavity, and may explain the origins of microstructure. The $m=0$ modes may correspond to the emission usually denoted as "core" (Rankin 1983; Lyne \& Manchester 1988), with the higher order $m$ modes yielding "conal" emission; indeed, for $m \approx 7-10$ various modes or combinations of modes have concentric rings of antinodes with a ratio of 
angular widths very close to those determined for the inner and outer cones of Rankin (1993).

The mode plots in Figure 1 can also be directly interpreted as polar emission maps, and show similarities to those of Deshpande \& Rankin (1999) and others. Furthermore, the polarization of the scattered emission is determined by the polarization of the underlying mode; since antinodes adjacent in the tube cross-section have orthogonal polarization this gives a direct mechanism for the observed orthogonal modes, while scattering of the $E_{\|}$component would tend to produce unpolarized emission, perhaps with some polarization in the plane of curvature of the magnetic field lines.

Provided that the modes are not pinned to structure in the tube's wall, the modes will be free to rotate about its axis and this will be observed as subpulse drift. For instance, this should be the case when the inclination angle $\chi$ between a pulsar's rotation and magnetic axes is small, making the tube's cross-section approximately circular; in turn this might explain why pulsars exhibiting quasistable subpulse drift generally have small $\chi$.

If it is also true that the modes are not pinned to the AC current beams, but rather that the current beams follow the mode antinodes, then the modes will rotate due to stellar rotation and general relativistic frame dragging. A standing wave can be viewed as a distribution of oscillators in space, all of which are synchronized, and to achieve this synchronization in some set of frames requires that $c^{-1} \oint\left(g_{0 \alpha} / g_{00}\right) d x^{\alpha}=0$. Then it is found that the modes, whatever their form, must rotate with a frequency $\left(1-2 G I / c^{2} r^{3}\right) \cos (\chi) P^{-1}$ where $r$ is the distance from the center of the star, and $I$ is the stellar moment of inertia. The sense of this rotation relative to the stellar surface is opposite to that of the stellar rotation at the rotational pole closest to the gap. This does not reproduce the long circulation times found by Deshpande \& Rankin (1999) and others and this failure will be discussed elsewhere. If frame-dragging and stellar rotation are not the cause of subpulse drift, then it suggests that the rotating modes are pinned to current density columns which circulate about the axis, probably due to $\mathbf{E} \times \mathbf{B}$-drift.

Acknowledgments. I thank R. Burman and R. Manchester for many useful suggestions, and UWA, the ATNF and my wife for financial support.

\section{References}

Deshpande, A. A., \& Rankin, J. M. 1999, ApJ, 524, 1008

Goldreich, P., \& Julian, W. H. 1969, ApJ, 157, 869

Harding, A. K., \& Muslimov, A. G. 1998, ApJ, 508, 328

Lyne, A. G., \& Manchester, R. N. 1988, MNRAS, 234, 477

Qiao, G. 1988, Vistas Astron., 31, 393

Rankin, J. M. 1983, ApJ, 274, 333

- 1993, ApJ, 405, 285

Ruderman, M. A., \& Sutherland, P. G. 1975, ApJ, 196, 51

Schumann, W. O. 1952, Z. Naturforsch., 7a, 149

Sturrock, P. A. 1971, ApJ, 164, 529 